\title{
Quality antenatal care services delivery at health facilities of Ethiopia, assessment of the structure/input of care setting
}

\author{
Atkure Defar ${ }^{1,2^{*}}$, Theodros Getachew ${ }^{3,4}$, Girum Taye ${ }^{3}$, Tefera Tadele ${ }^{1}$, Misrak Getnet ${ }^{1}$, Tigist Shumet ${ }^{3}$, \\ Gebeyaw Molla ${ }^{3}$, Geremew Gonfa ${ }^{3}$, Habtamu Teklie ${ }^{1}$, Ambaye Tadesse $^{3}$ and Abebe Bekele ${ }^{5}$
}

\begin{abstract}
Background: According to the Donabedian model, the assessment for the quality of care includes three dimensions. These are structure, process, and outcome. Therefore, the present study aimed at assessing the structural quality of Antenatal care (ANC) service provision in Ethiopian health facilities.

Methods: Data were obtained from the 2018 Ethiopian Service Availability and Readiness Assessment (SARA) survey. The SARA was a cross-sectional facility-based assessment conducted to capture health facility service availability and readiness in Ethiopia. A total of 764 health facilities were sampled in the 9 regions and 2 city administrations of the country. The availability of equipment, supplies, medicine, health worker's training and availability of guidelines were assessed. Data were collected from October-December 2017. We run a multiple linear regression model to identify predictors of health facility readiness for Antenatal care service. The level of significance was determined at a $p$-value $<0.05$.
\end{abstract}

Result: Among the selected health facilities, $80.5 \%$ of them offered Antenatal care service. However, the availability of specific services was very low. The availability of tetanus toxoid vaccination, folic acid, iron supplementation, and monitoring of hypertension disorder was, $67.7,65.6,68.6$, and $75.1 \%$, respectively. The overall mean availability among the ten tracer items that are necessary to provide quality Antenatal care services was $50 \%$. In the multiple linear regression model, health centers, health posts and clinics scored lower Antenatal care service readiness compared to hospitals. The overall readiness index score was lower for private health facilities $(\beta=-0.047,95 \% \mathrm{Cl}$ : $(-0.1,-0.004)$. The readiness score had no association with the facility settings (Urban/Rural) ( $p$-value $>0.05)$. Facilities in six regions except Dire Dawa had $(\beta=0.067,95 \% \mathrm{Cl}:(0.004,0.129)$ lower readiness score than facilities in Tigray region ( $p$-value $<0.015$ ).

Conclusion: This analysis provides evidence of the gaps in structural readiness of health facilities to provide quality Antenatal care services. Key and essential supplies for quality Antenatal care service provision were missed in many of the health facilities. Guaranteeing properly equipped and staffed facilities shall be a target to improve the quality of Antenatal care services provision.

Keywords: Antenatal care, Ethiopia, Quality, Structure, Input, Readiness score

\footnotetext{
* Correspondence: atkuredefar@gmail.com

${ }^{1}$ Reproductive Health Research Team, Ethiopian Public Health Institute, Addis Ababa, Ethiopia

2Department of Epidemiology and Biostatistics, University of Gondar, College of Medicine and Health Science, Institute of Public health, Gondar, Ethiopia Full list of author information is available at the end of the article
}

(c) The Author(s). 2020 Open Access This article is licensed under a Creative Commons Attribution 4.0 International License, which permits use, sharing, adaptation, distribution and reproduction in any medium or format, as long as you give appropriate credit to the original author(s) and the source, provide a link to the Creative Commons licence, and indicate if changes were made. The images or other third party material in this article are included in the article's Creative Commons licence, unless indicated otherwise in a credit line to the material. If material is not included in the article's Creative Commons licence and your intended use is not permitted by statutory regulation or exceeds the permitted use, you will need to obtain permission directly from the copyright holder. To view a copy of this licence, visit http://creativecommons.org/licenses/by/4.0/ The Creative Commons Public Domain Dedication waiver (http://creativecommons.org/publicdomain/zero/1.0/) applies to the data made available in this article, unless otherwise stated in a credit line to the data. 


\section{Background}

In a focused ANC program, four visits are required to guarantee the safety of a woman and her newborn through the provision of essential interventions [1]. However, in a revised World Health Organization guideline a minimum of eight ANC contacts is recommended in the absence of complications [2]. Therefore, to respond this need, health facilities should be well prepared in terms of trained human power, drugs, supplies, equipment and infrastructure.

Antenatal care is also an opportunity to promote the use of skilled attendance at birth and healthy behaviours such as breastfeeding, early postnatal care and planning for optimal pregnancy spacing [3]. Different community and facility-based studies have proved the negative effect of low Antenatal care service utilization and readiness of health facilities on the continuum of care, which flagged for a comprehensive program and research conduct [4].

Overall, to assess the service provision of Antenatal care services, four key components are required to be measured. These are; iron supplementation, tetanus toxoid vaccination, folic acid supplementation and monitoring for pregnancy-related hypertension disorder [5]. However, management of the Obstetric complications (such as preeclampsia, eclampsia, haemorrhage, tetanus toxoid immunization, and intermittent preventive treatment for malaria during pregnancy (IPTp) are among the essential intervention services that are required to be available at health facilities. The provision of these services should also be extended to the identification and management of infections [6].

However, it has shown negative improvement overtime in recent years with disparities by regions and facility types [7]. The recent Ethiopian Emergency Obstetric and Newborn Care assessment revealed that majority of health care facilities offered Antenatal service and not varied by regions, health facility type and ownership [8].

There were very few studies that assessed the quality of care for Antenatal care services [9-11]. These studies included few health facilities in limited geographic areas, focused on perceived quality of care and they assessed only factors associated with client perspective but not at facility level.

The availability and readiness of services are important factors that determine the health care service utilization [12]. However, it can not substitute service quality. Thus, measuring what is being delivered, rather than focusing on how often care is delivered is more important $[10,13]$.

Different models help to measure the quality of care provision [14]. Of these, the current study used Donabedian quality of care framework to measure structural quality. This study was the first of its kind to assess quality of Antenatal care service at the national level using structural indices. Therefore, this study aimed to assess the service availability, structural readiness of facilities and associated factors to provide quality Antenatal care service.

\section{Method}

\section{Study area and data source}

The Ethiopian health system has three tiers of service having more than 24,000 health facilities of different types [15]. The majority of services are provided at the primary level, which is organized around one primary hospital serving four or five Primary Health Care Units (PHCUs). As indicated in Fig. 1, a PHCU comprises a Primary hospital, health center and five satellite health posts. These serve as an approximate of 100, 000, 25,000 and 5000 people, respectively. A primary hospital is a referral center for the catchment area of the health center. General hospitals are the second level in health care tier system. These hospitals are referral centers for primary hospitals and provide service of approximately one million people. The tertiary level is specialized hospitals that serves about 5 million people [16].

The Ethiopian health service is augmented by the rapid expansion of the private for-profit and NonGovernmental Organization's facilities that play a significant role in the health care system. They are concentrated largely in urban areas. However, the public sector delivers most of the health services in the country. This enhanced the Public-Private/NGO partnership and supported the health care services provided in the country.

Ethiopian has conducted Service Availability and Rreadiness Assessemnt in 2016 for the first time and recently in 2018. The present study used data from the 2018 SARA survey. The data was collected at a national level in all the nine regional states and two city administration (namely; Tigray, Afar, Amhara, Oromia, Somali, Benishangul-Gumuz, SNNP, Gambella, Harari, and two city administrations; i.e. Addis Ababa and Dire Dawa). The study was conducted from October to December 2017.

\section{Study design and sampling methods}

A cross-sectional study design was conducted among selected health facilities in Ethiopia. The sample allocation considered the skewed regional level distribution of Ethiopian health facility. We used a stratified sampling technique. The stratification was based on facility type and managing authority. All hospitals, selected health centers, clinics and health posts were included in the sample. All health facilities that were included in the 2016 SARA were part of the 2018 SARA study [7] and included in the current analysis. New hospitals that were 


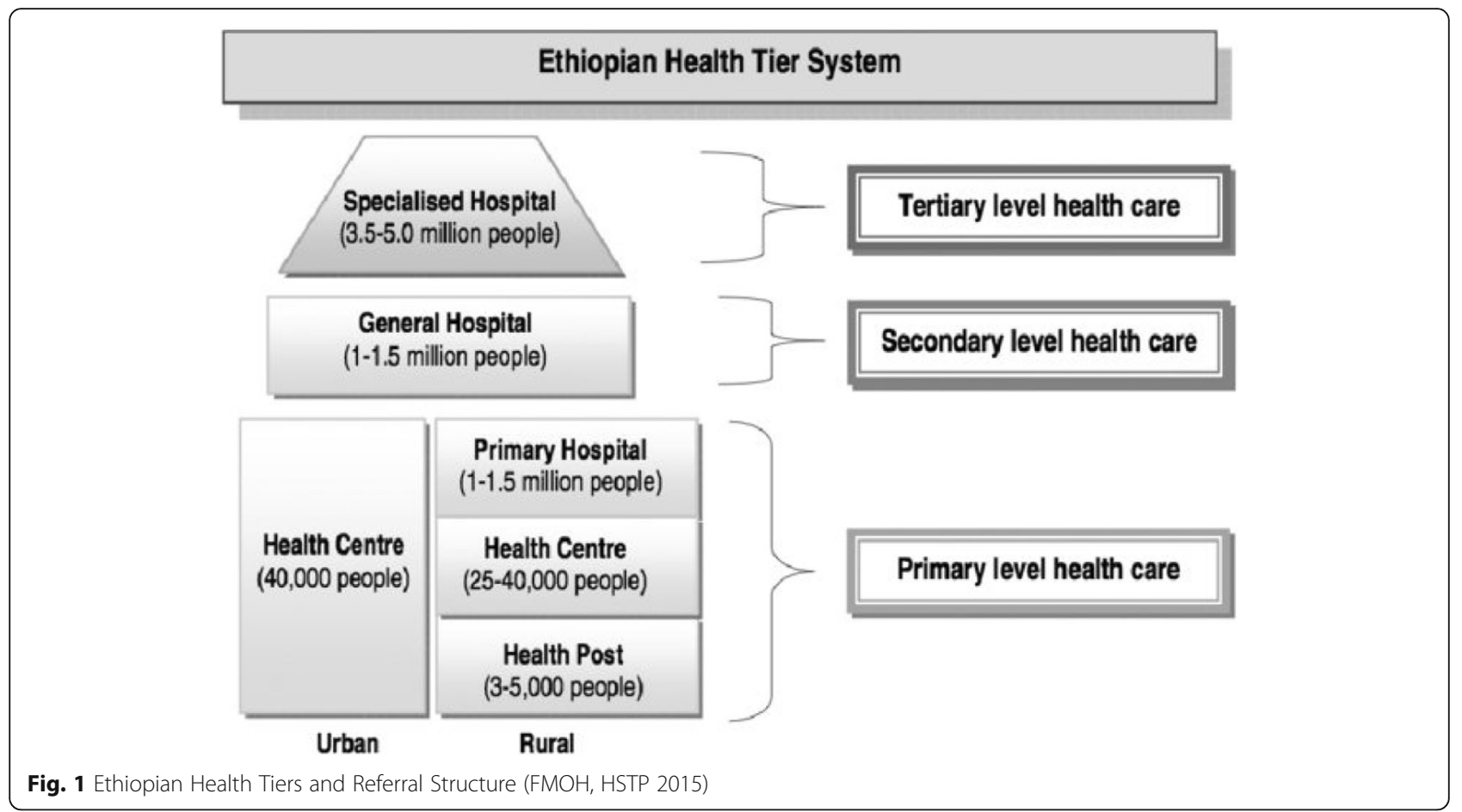

constructed afterwards in 2016 were included in the 2018 SARA survey.

\section{Sample size}

The sample size was estimated using the following formula; $n=[[(\mathrm{z} 2 * \mathrm{p} * \mathrm{q})+\mathrm{ME} 2] /[\mathrm{ME} 2+\mathrm{z} 2 * \mathrm{p} * \mathrm{q} / \mathrm{N}]] * \mathrm{~d}$,

Where $n=$ the sample size, $\mathrm{z}=$ the square of the normal deviate at the required confidence level (3.84 is the square of the normal deviate (1.96) needed to provide an estimate at the $95 \%$ level of confidence) $p=$ the proportion of facilities with the attribute of interest (A $47 \%$ of the Proportion of facilities with basic amenities from the 2016 SARA result was used), $q=1-p, M E=$ margin of error (15\%), $d=$ the design effect (because of the regional stratification we assumed a design effect of 1.5). $N=$ Total number of Facilities in each stratum. Basic Amenities was defined as the mean availability of seven basic amenities, expressed in percentage. The items were; Electric power, Improved water source, Room with privacy, Adequate sanitation facilities, Communication equipment, Access to computer with internet, and Emergency transportation. The sample size was required to provide a national representation within $95 \%$ confidence level and $+/-15 \%$ precision. The sampling considered $20 \%$ for private clinics and additional $10 \%$ for refusals or closed facilities.

Thus, a total of 764 facilities were included in the study; 164 health centers, 165 clinics (Private facilities; either lower, medium or higher clinics) and 132 health posts. This number was within the range of 500 to 800 facilities of the World Health Organization recommendations for Service Availability and Readiness Assessment surveys that is required to have regional estimates (Table 1).

\section{Data collection tool}

The survey used computer-based electronic data collection using the Census and Survey Processing System (CSPro) data entry entry template. The data collection instruments were adopted from the WHO annual health facility monitoring guide [17]. Information on the health facility infrastructure; facility audit questions focused on the availability of trained personnel, clinical and laboratory services, medicines, and guidelines, information of location, and functional status components of support systems were collected. The measurement of the service availability and readiness of the ANC was made on four main components. These were; Iron Supplementation, Folic Acid supplementation, Tetanus Toxoid vaccination and Monitoring for the hypertensive disorder of pregnancy.

\section{Outcome variables}

We measured the structural quality of care for Antenatal care service. To measure this, we used a combination of 10 trace items that are necessary to provide quality antenatal care service. These were availability of trained personnel in the past 2-years, ANC checklist and/or job 
Table 1 Distribution of the study facilities by region, facility type and managing authority

\begin{tabular}{|c|c|c|c|c|c|}
\hline Characteristics & Hospital & Health center & Health Post & Clinics & Total \\
\hline Region & $n(\%)$ & $\mathrm{n}(\%)$ & $\mathrm{n}(\%)$ & $\mathrm{n}(\%)$ & $n(\%)$ \\
\hline Tigray & $39(12.9)$ & $14(8.5)$ & $11(8.3)$ & $12(7.3)$ & 76(9.9) \\
\hline Afar & $6(2.0)$ & 16(9.8) & $12(9.1)$ & 15(9.1) & $49(6.4)$ \\
\hline Amhara & $65(21.5)$ & $17(10.4)$ & $16(12.1)$ & $16(9.7)$ & $114(14.9)$ \\
\hline Oromia & $75(24.8)$ & $16(9.8)$ & $16(12.1)$ & 18(10.9) & $125(16.4)$ \\
\hline Somali & $11(3.6)$ & 16(9.8) & $13(9.8)$ & $17(10.3)$ & $57(7.5)$ \\
\hline Benishangul Gumuz & $2(0.7)$ & $15(9.1)$ & $14(10.6)$ & $14(8.5)$ & $45(5.9)$ \\
\hline S.N.N.P & $58(19.1)$ & $16(9.8)$ & $16(12.1)$ & 15(9.1) & 105(13.7) \\
\hline Gambella & $3(1.0)$ & $13(7.9)$ & $12(9.1)$ & $14(8.5)$ & $42(5.5)$ \\
\hline Harari & $5(1.7)$ & $8(4.9)$ & $11(8.3)$ & $11(6.7)$ & $35(4.6)$ \\
\hline Addis Ababa & $32(10.6)$ & $22(13.4)$ & $0(0.0)$ & 23(13.9) & $77(10.1)$ \\
\hline Dire Dawa & $7(2.3)$ & $11(6.7)$ & $11(8.3)$ & $10(6.1)$ & $39(5.1)$ \\
\hline \multicolumn{6}{|l|}{ Managing Authority } \\
\hline Public & $243(80.2)$ & 160(97.6) & $132(100)$ & $7(4.2)$ & $542(70.9)$ \\
\hline Others & $60(19.8)$ & $4(2.4)$ & $0(0)$ & 158(95.8) & $222(29.1)$ \\
\hline \multicolumn{6}{|l|}{ Location } \\
\hline Urban & 273(90.1) & $86(52.4)$ & $10(7.6)$ & 135(81.8) & $504(66.0)$ \\
\hline Rural & 30(9.9) & 78(47.6) & $122(92.4)$ & $30(18.2)$ & $260(34.0)$ \\
\hline Total & 303 & 164 & 132 & 165 & 764 \\
\hline
\end{tabular}

aid, Guideline on ANC service, and Availability of supplies and drugs (availability of Hemoglobin, Urine dipstick, Iron Tablets, Folic Acid, Tetanus toxoid Vaccine, Intermittent Preventive Treatment in pregnancy, and Insecticide Treated bed Net. As a secondary outcome, we measured the overall service availability of ANC and components of antennal care service. Such as; Iron supplementation, Folic acid supplementation, Tetanus toxoid vaccination, and Monitoring for hypertensive disorder of pregnancy.

\section{Explanatory variables}

Explanatory variables were; region, facility type, managing authority and facility setting (Urban/Rural).

\section{Data management and analysis}

We used the WHO reference manual to select the structural readiness score items [17]. The items were counted for each case and added up to one number to calculate the mean. This gave us a continuous variable ranging from 0 to 10 as an outcome of interest.

We used multiple linear regression model to determine the association between the overall index for quality of care and region, facility type, ownership, and facility setting. The SPSS version 20 and STATA version 14 software's were used to do the analysis and ArcGIS version 10.4 was used to produce the map.

\section{Results}

\section{Antenatal care service availability and readiness}

The availability of ANC service was assessed using four components of the service. These were; Iron supplementation, folic acid supplementation, tetanus toxoid vaccination and monitoring for the hypertensive disorder of pregnancy. Antenatal care service was not offered in almost one in five of the surveyed facilities. Iron supplementation was available in $68.6 \%$ of the surveyed health facilities followed by Tetanus toxoid vaccination, that was $67.7 \%$. Three-fourth and nearly two-thirds of surveyed facilities have provided monitoring for the hypertensive disorder of pregnancy and folic acid supplementation, respectively.

There was regional variation in offering ANC services. Higher than $89 \%$ of facilities in Tigray and Amhara regions provided ANC service, while the lowest (57.1\%) was in Gambella. Antenatal care services were universally offered in general hospitals, primary hospitals and health centers. Whereas, only 28 and $29 \%$ of medium and lower clinics offered the service, respectively. In addition, tetanus toxoid vaccination offered in only 7 and $4 \%$ of the medium and lower clinics, respectively.

Ninety-three percent of the government-owned facilities provided ANC service. The availability of ANC service was almost similar in both rural and urban health facilities. Seventy-eight percent of the facilities in Tigray 
and Afar offered iron supplementation, whereas the provision of monitoring of the hypertension disorder for pregnancy was highest in Tigray's health facilities compared with other regions (Table 2 and Fig. 2).

Table 3 depicted the distribution of tracer items among facilities that offered ANC service. Accordingly, the mean availability of tracer items was highest in hospitals and health centers than other facility types. On average, clinics and health posts had three tracer items out of ten that are necessary to provide ANC service.

The availability of tracer items for the provision of ANC service in the hospital ranged from the highest, $98.7 \%$ for blood pressure apparatus and lowest to $14 \%$ for Insecticide Treated Nets (ITNs). Blood pressure apparatus was the most available $(96.3 \%)$ tracer item in health centers followed by ANC check-lists and/or jobaids (81.5\%). ITNs was the least available (29.5\%) tracer item in health centers. However, the availability was higher among the health center than any other type of health facilities. Fifty-two percent the health post had Blood pressure apparatus, followed by checklists and/or job-aids (50\%) and Iron tablets (49\%), Blood pressure apparatus was the most available ANC service-related item in clinics (98\%) while the least was ITNs, that was null. There were no kits for Haemoglobin and urine dipstick test in health posts (Table 3 ).

Table 2 Percentage of facilities with ANC care and service by, regions, facility type, setting, $n=764$

\begin{tabular}{|c|c|c|c|c|c|c|c|c|c|c|c|}
\hline \multirow[t]{3}{*}{ Facility Characteristics } & \multirow{2}{*}{\multicolumn{2}{|c|}{$\begin{array}{l}\text { Offers } \\
\text { antenatal care }\end{array}$}} & \multicolumn{4}{|c|}{ Supplementation } & \multirow{2}{*}{\multicolumn{2}{|c|}{$\begin{array}{l}\text { Tetanus toxoid } \\
\text { vaccination }\end{array}$}} & \multirow{2}{*}{\multicolumn{2}{|c|}{$\begin{array}{l}\text { Monitoring for } \\
\text { hypertensive } \\
\text { disorder of } \\
\text { pregnancy }\end{array}$}} & \multirow{3}{*}{$\begin{array}{l}\text { Total } \\
\text { number } \\
\text { of } \\
\text { facilities } \\
\text { (a UW) } \\
\text { n }\end{array}$} \\
\hline & & & \multicolumn{2}{|l|}{ Iron } & \multicolumn{2}{|c|}{ Folic acid } & & & & & \\
\hline & $n$ & $\%$ & $\mathrm{n}$ & $\%$ & $\mathrm{~N}$ & $\%$ & $n$ & $\%$ & $n$ & $\%$ & \\
\hline \multicolumn{12}{|l|}{ Region } \\
\hline Tigray & 69 & 90.8 & 59 & 77.6 & 58 & 76.3 & 55 & 72.4 & 65 & 85.5 & 76 \\
\hline Afar & 38 & 77.6 & 38 & 77.6 & 35 & 71.4 & 31 & 63.3 & 37 & 75.5 & 49 \\
\hline Amhara & 101 & 88.6 & 86 & 75.4 & 84 & 73.7 & 79 & 69.3 & 94 & 82.5 & 114 \\
\hline Oromia & 106 & 84.8 & 92 & 73.6 & 86 & 68.8 & 90 & 72.0 & 96 & 76.8 & 125 \\
\hline Somali & 39 & 68.4 & 34 & 59.6 & 34 & 59.6 & 33 & 57.9 & 35 & 61.4 & 57 \\
\hline Benishangul -Gumuz & 35 & 77.8 & 26 & 57.8 & 27 & 60.0 & 29 & 64.4 & 30 & 66.7 & 45 \\
\hline S.N.N.P & 91 & 86.7 & 77 & 73.3 & 66 & 62.9 & 83 & 79.0 & 86 & 81.9 & 105 \\
\hline Gambella & 24 & 57.1 & 19 & 45.2 & 18 & 42.9 & 21 & 50.0 & 22 & 52.4 & 42 \\
\hline Harari & 27 & 77.1 & 24 & 68.6 & 24 & 68.6 & 22 & 62.9 & 26 & 74.3 & 35 \\
\hline Addis Ababa & 56 & 72.7 & 47 & 61.0 & 48 & 62.3 & 48 & 62.3 & 54 & 70.1 & 77 \\
\hline Dire Dawa & 29 & 74.4 & 22 & 56.4 & 21 & 53.8 & 26 & 66.7 & 29 & 74.4 & 39 \\
\hline \multicolumn{12}{|l|}{ Facility type } \\
\hline Referral Hospital & 29 & 93.5 & 27 & 87.1 & 27 & 87.1 & 28 & 90.3 & 29 & 93.5 & 31 \\
\hline General Hospital & 116 & 100 & 103 & 88.8 & 100 & 86.2 & 96 & 82.8 & 115 & 99.1 & 116 \\
\hline Primary Hospital & 154 & 98.7 & 139 & 89.1 & 132 & 84.6 & 134 & 85.9 & 152 & 97.4 & 156 \\
\hline Health Center & 162 & 98.8 & 141 & 86.0 & 136 & 82.9 & 158 & 96.3 & 157 & 95.7 & 164 \\
\hline Health Post & 104 & 78.8 & 84 & 63.6 & 75 & 56.8 & 87 & 65.9 & 74 & 56.1 & 132 \\
\hline Higher Clinic & 8 & 42.1 & 6 & 31.6 & 6 & 31.6 & 6 & 31.6 & 8 & 42.1 & 19 \\
\hline Medium Clinic & 21 & 28.4 & 14 & 18.9 & 14 & 18.9 & 5 & 6.8 & 19 & 25.7 & 74 \\
\hline Lower Clinic & 21 & 29.2 & 10 & 13.9 & 11 & 15.3 & 3 & 4.2 & 20 & 27.8 & 72 \\
\hline \multicolumn{12}{|l|}{ Managing Authority } \\
\hline Public & 503 & 92.8 & 438 & 80.8 & 417 & 76.9 & 460 & 84.9 & 465 & 85.8 & 542 \\
\hline Others & 112 & 50.5 & 86 & 38.7 & 84 & 37.8 & 57 & 25.7 & 109 & 49.1 & 222 \\
\hline \multicolumn{12}{|l|}{ Facility setting } \\
\hline Urban & 404 & 80.2 & 341 & 67.7 & 333 & 66.1 & 333 & 66.1 & 395 & 78.4 & 504 \\
\hline Rural & 211 & 81.2 & 183 & 70.4 & 168 & 64.6 & 184 & 70.8 & 179 & 68.8 & 260 \\
\hline Total number of facilities ${ }^{a}$ & 615 & 80.5 & 524 & 68.6 & 501 & 65.6 & 517 & 67.7 & 574 & 75.1 & 764 \\
\hline
\end{tabular}




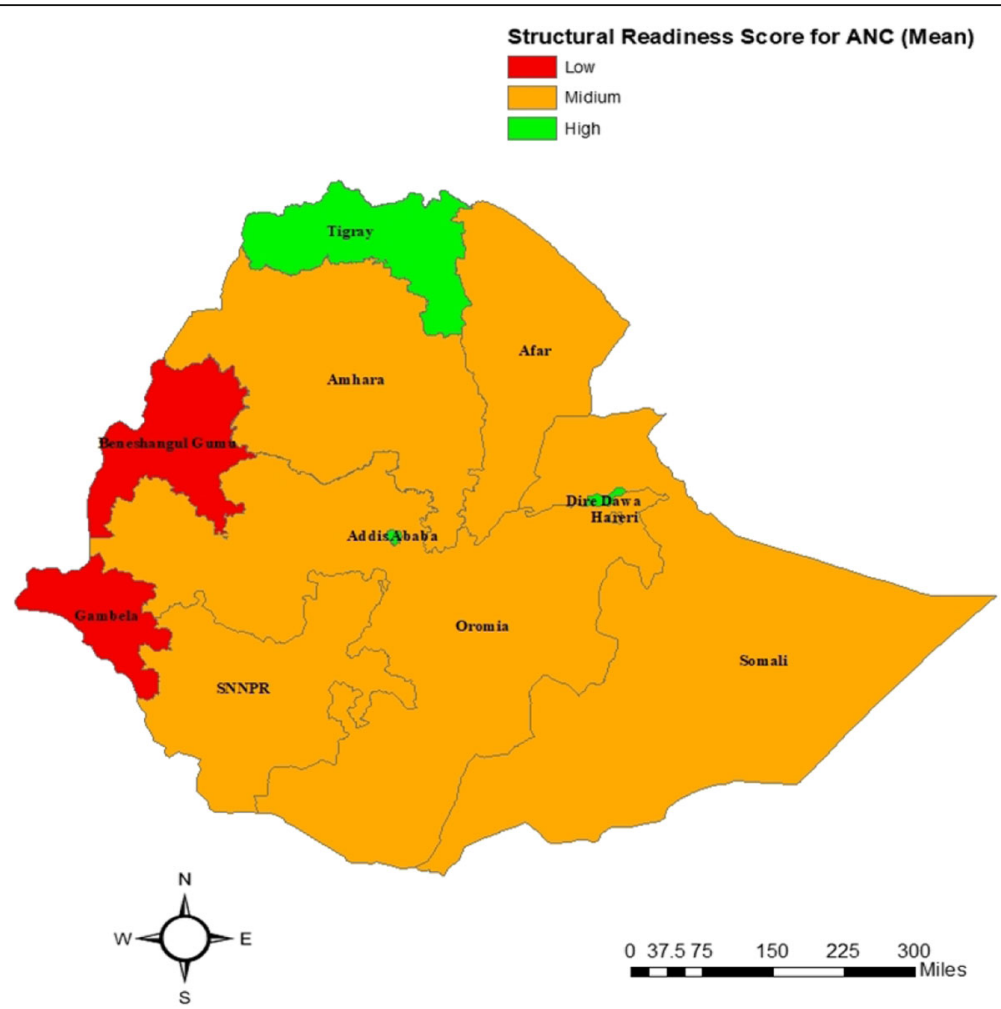

Fig. 2 Maps showing the structural readiness score for Antenatal care by region in Ethiopia. (The authors produced the map and acknowledge the software (ArcGIS) used in the data management and analysis section)

\section{Determinants of structural quality of ANC services}

A linear regression model was used to check the association between facility type, managing authority, setting, and region with the readiness score to provide quality Antenatal care services. Accordingly, at 5\% level of significance, health centers had $(\beta=-0.083$,
95\%CI: $-0.119,-0.047)$, health posts $(\beta=-0.383$, 95\% CI: $-0.427,-0.338)$, and clinics $(\beta=-0.389$, 95\%CI: - 0.434, - 0.344) lower readiness score compared with hospitals. The overall structural readiness score for quality ANC was lower for private health facilities $(\beta=-0.047,95 \% \mathrm{CI}:(-0.1,-0.004)$ than that

Table 3 Distribution of tracer items by facility type, $n=615$

\begin{tabular}{|c|c|c|c|c|c|c|c|c|c|c|}
\hline \multirow{2}{*}{\multicolumn{2}{|c|}{ Tracer items measuring structural quality }} & \multicolumn{2}{|c|}{ Hospital } & \multicolumn{2}{|c|}{ Health center } & \multicolumn{2}{|c|}{ Health Post } & \multicolumn{2}{|c|}{ Clinics } & \multirow{2}{*}{$\begin{array}{l}\text { Total } \\
\mathrm{n}\end{array}$} \\
\hline & & $n$ & $\%$ & $\mathrm{n}$ & $\%$ & $\mathrm{n}$ & $\%$ & $\mathrm{n}$ & $\%$ & \\
\hline \multicolumn{2}{|c|}{ Guidelines available for antenatal care service } & 137 & 45.8 & 76 & 46.9 & 29 & 27.9 & 11 & 22.0 & 253 \\
\hline \multicolumn{2}{|c|}{ ANC check-lists and/or job-aids } & 235 & 78.6 & 132 & 81.5 & 52 & 50.0 & 23 & 46.0 & 442 \\
\hline \multicolumn{2}{|c|}{ At least one trained staff for antenatal care service } & 120 & 40.1 & 57 & 35.2 & 36 & 34.6 & 14 & 28.0 & 227 \\
\hline \multicolumn{2}{|c|}{ Blood pressure apparatus } & 295 & 98.7 & 156 & 96.3 & 54 & 51.9 & 49 & 98.0 & 554 \\
\hline \multicolumn{2}{|l|}{ Haemoglobin test } & 170 & 56.9 & 53 & 32.7 & 0 & 0 & 19 & 38.0 & 242 \\
\hline \multicolumn{2}{|c|}{ Urine dipstick protein test } & 287 & 96.0 & 111 & 68.5 & 0 & 0 & 27 & 54.0 & 425 \\
\hline \multicolumn{2}{|l|}{ Iron tablets } & 244 & 81.6 & 115 & 71.0 & 51 & 49.0 & 15 & 30.0 & 425 \\
\hline \multicolumn{2}{|l|}{ Folic acid tablets } & 251 & 83.9 & 117 & 72.2 & 49 & 47.1 & 13 & 26.0 & 430 \\
\hline \multicolumn{2}{|c|}{ Tetanus toxoid vaccine } & 205 & 68.6 & 119 & 73.5 & 32 & 30.8 & 6 & 12.0 & 362 \\
\hline \multicolumn{2}{|l|}{ ITNs } & 41 & 13.7 & 48 & 29.6 & 29 & 27.9 & 0 & 0.0 & 118 \\
\hline \multirow[t]{2}{*}{ Tracer item score } & Mean & 6.6 & & 6.1 & & 3.2 & & 3.5 & & 5.7 \\
\hline & SE & 0.1 & & 0.1 & & 0.2 & & 0.3 & & 0.1 \\
\hline \multicolumn{2}{|c|}{ Total facilities (Unweighted) } & 299 & & 162 & & 104 & & 50 & & 615 \\
\hline
\end{tabular}


of public health facilities and did not association with the locations of facilities ( $p$-value $>0.05$ ) (Table 4).

There was regional difference in structural readiness to provide quality ANC services. Facilities in Amhara ( $\beta=$ -0.132 , 95\%CI: $(-0.179,-0.085))$, Oromia $(\beta=-0.152$, 95\%CI: $(-0.198,-0.106)$, Benishangul Gumuz $(\beta=-.132$, 95\%CI: $(-0.178,-0.057)$, SNNP. $(\beta=-0.161,95 \%$ CI: $(-0.209,-0.115)$, Gambella $(\beta=-0.15,95 \%$ CI: $(-0.212$, $-0.089)$, Addis Ababa $(\beta=-0.066,95 \% \mathrm{CI}:(-0.12$, $-0.013)$ regions had lower overall readiness score for ANC service than facilities in the Tigray region $(p$-value $<0.05)$. However, the overall ANC service readiness score for Dire Dawa city $(\beta=0.067$, 95\%CI: $(0.004,0.129)$ was higher than that of the Tigray region. The variables included in the model explained $60 \%$ of the variability $\left(R^{2}=0.6\right)$.

\section{Discussion}

The availability of Antenatal care service was assessed using four components of the service. More than eight out of ten surveyed health facilities offered ANC service. The availability of the four components of ANC services was ranging from 65 to $75 \%$. In overall, the mean availability of the ten tracer items that are necessary to provide quality ANC service was less than six. Hospitals were by far better in the provision of quality ANC service than other health facility types. Health centers, health posts and clinics had lower overall readiness score than hospitals. The overall readiness index score for private health facilities was lower than the public and had no difference by location of facilities. There was regional variation in readiness score.

In contrary to the current study, a study that used data from 20 nationally representative health facility assessments of both the SPA and the SARA revealed a higher proportion of health facility readiness to deliver iron supplementation (84.9\%), tetanus toxoid vaccination (82.8\%) and low result for hypertensive disease case management (23.0\%) [18].

Our study demonstrated that there was a critical gap (40\%) of health care providers who have been trained to provide ANC service. Congruently, the study conducted in Ugandan has shown an overall staffing gap of over $40 \%$ [19]. As the availability of trained health care

Table 4 Factors associated with structural readiness score of ANC service provision

\begin{tabular}{|c|c|c|c|c|c|c|}
\hline \multirow{2}{*}{$\begin{array}{l}\text { Facility characteristics } \\
\text { Facility type }\end{array}$} & \multirow{2}{*}{$\begin{array}{l}\text { ANC } \\
\text { Readiness } \\
\text { Score } \\
\text { index }\end{array}$} & \multirow[t]{2}{*}{ SE } & \multicolumn{2}{|c|}{ Crude Model } & \multicolumn{2}{|c|}{ Adjusted Model } \\
\hline & & & B & $95 \% \mathrm{Cl}$ & B & $95 \% \mathrm{Cl}$ \\
\hline Hospital (Ref) & 6.6 & .660 & & & & \\
\hline Health center & 6.1 & .017 & -.056 & $(-.089,-.023)$ & -.083 & $(-.119,-.047)$ \\
\hline Health post & 3.2 & .018 & -.368 & $(-.403,-.332)$ & -.383 & $(-.427,-.338)$ \\
\hline Clinics $^{a}$ & 3.5 & .017 & -.398 & $(-.431,-.365)$ & -.389 & $(-.434,-.344)$ \\
\hline \multicolumn{7}{|l|}{ Managing authority } \\
\hline Public (Ref) & 5.8 & .019 & .01 & & & \\
\hline Others & 5.1 & .552 & -.019 & $(-.221,-.147)$ & -.047 & $(-.1-.004)$ \\
\hline \multicolumn{7}{|l|}{ Facility setting } \\
\hline Urban (Ref) & 6.2 & .011 & & & & \\
\hline Rural & 4.7 & .018 & -.136 & $(-.172,-.100)$ & -.026 & $(-.058, .006)$ \\
\hline \multicolumn{7}{|l|}{ Region } \\
\hline Tigray (Ref) & 6.7 & .028 & & & & \\
\hline Afar & 5.3 & .044 & -.154 & $(-.241,-.068)$ & -.039 & $(-.097, .019)$ \\
\hline Amhara & 5.6 & .036 & -.119 & $(-.189,-.049)$ & -.132 & $(-.179,-.085)$ \\
\hline Oromiya & 5.5 & .035 & -.136 & $(-.204,-.067)$ & -.152 & $(-.198,-.106)$ \\
\hline Somali & 5.8 & .042 & -.129 & $(-.212,-.046)$ & -.029 & $(-.085, .026)$ \\
\hline Benishangul Gumuz & 4.2 & .045 & -.263 & $(-.352,-.174)$ & -.117 & $(-.178,-.057)$ \\
\hline S.N.N.P. & 5.2 & .036 & -.156 & $(-.227,-.085)$ & -.161 & $(-.209,-.115)$ \\
\hline Gambela & 4.2 & .046 & -.297 & $(-.387,-.205)$ & -.150 & $(-.212,-.089)$ \\
\hline Harari & 5.9 & .049 & -.119 & $(-.215,-.023)$ & .019 & $(-.047, .084)$ \\
\hline Addis Ababa & 6.6 & .039 & -.083 & $(-.160,-.007)$ & -.066 & $(-.12,-.013)$ \\
\hline Dire Dawa & 6.5 & .047 & -.04 & $(-.134, .053)$ & .067 & $(.004, .129)$ \\
\hline
\end{tabular}

${ }^{a}$ Clinic: include higher, medium and lower clinics 
providers is one of the strategies to improve the availability and quality of the service being provided, there should be a mechanism to overcome shortage of trained staff.

One of the determinant factors for the provision of quality of antenatal care service is the availability of equipment. In this regard, the current study has discovered that Ethiopian health facilities were poorly equipped. Similarly, a study conducted in Nigeria and Uganda showed as the quality of care was determined by the availability of medical equipment $[19,20]$. If the measurement on the availability of equipment included the working status or the functionality, the result could have been different. Thus, to get the true picture of the quality of the service provided, assessments should consider the working status of the equipment.

A study conducted by Taddese and his collogues on quality of antenatal care services at public health facilities of Northwest of Ethiopia described that there was a lack of diagnostic reagents which was partly explained the problems observed in the provision of recommended antenatal care [21]. Similarly, in this study, the availability of diagnostic service for ANC was low and affected the overall readiness score.

The current study depicted the regional variation on service availability for ANC. Similarly, a multi-country study that used data from the Demographic and Health Survey has revealed the existence of geographic variation of ANC service readiness [22]. However, we did not observe any significant difference by facilities settings (Urban/Rural).

The overall ANC readiness score was fonnd to be low, which was 5.7. However, a study conducted in india had measured quality of ANC care in different ways using the clinical quality, interpersonal, quality of care and utilization of antennal care measures, likewise, It has found low quality of Antenatal care in the surveyed health facilities [23]. Contradicting to this, a study conducted to assess the quality of antenatal and childbirth care in selected rural health facilities in Burkina Faso, Ghana and Tanzania revealed a satisfactory level quality of Antenatal care service [24]. The study used the availability of infrastructure, essential equipment, essential drugs, vaccines and laboratory supplies as a measure of quality. However, to measure the structural readiness we used a WHO guideline for the assessment of health service availability and readiness. We have found that public health facilities were better in readiness to provide ANC service. Congruent with our findings, a study conducted in Vietnam and India has found that public health facilities were superior in readiness to that of the private health facilities [25, 26]. Moreover, our study finding was evinced by a multi-country study that was conducted in 46 Low and Middle-Income Countries
[22]. The study reported that public health facilities were the main source of ANC services.

Findings from this study should be considered in the context of some limitations. Failure to compare and associate with many studies conducted in Ethiopia was one of the weaknesses of this study. This was due to the type and size of the data used. Besides, this study did not capture provider-level data that would tell more about the quality of care from the provider's perspective. Thus, using a qualitative study is more helpful to understand the quality of care in the perspective of health care provider's. The study has several strengths. We did the analysis using nationally representative data and we were able to generate evidence at regional level to show the geographic variability, which indirectly inform the service equity. In addition, unlike most available studies, this study focuses on showing facility-level structural quality.

\section{Conclusion}

The overall readiness score to provide ANC service was low in Ethiopian health facilities. Majority of the health facilities lacks the key components of Antenatal care service. There was a difference in overall readiness of ANC service provision by region, facility type and ownership of the facility. In general, the availability and readiness of ANC service at health posts and clinics were below the expected. Addressing the regional disparity and provision of training for health care providers were found to be vital to improve the quality of service provision. As the quality and equity of maternal care are the core agenda of the health sector, attention shall be given to improve ANC service provision through proper resource allocation. Ensuring the availability of trained health workers, equipment, drugs and guidelines for Antennal care are the cornerstones for continuous improvement of facility-based health care services. Policymakers, program personnel, and partners shall use this evidence for the improvement of health service delivery. Further program evaluations and implementation researches are highly recommended to understand the multi-dimensional nature of the quality of care.

\footnotetext{
Abbreviations

CSPro: Census and Survey Processing System; IRB: Institutional Review Board; ITNs: Insecticides Treated Nets; LMICs: Low and Middle-Income Countries; IPT: Intermittent Preventive Treatment for Pregnancy; SARA : Service Availably and Readiness Assessment; ESPA+: Ethiopian Service Provision Assessment plus
}

\section{Acknowledgements}

Authors would like to thank all stockholder who has been participated financially or technically. We also would like to thank regional health bureaus, district health offices for their smooth and kind cooperation. Special thanks to all the field staff for their excellent fieldwork. 


\section{Authors' contributions}

AD conceptualized the study, data analysis, and wrote the paper. TG mainly supports data analysis and write-up. GT, TT, MG, GM, HT, GG, AT and TS wrote some parts of the paper. AB critically reviewed the manuscript and approved. All authors participated in the acquisition, analysis, and interpretation of the data. Finally, all authors read and approved the final manuscript.

\section{Funding}

Fund has been received from WHO in the design of the study, collection, analysis, and interpretation of SARA project. However, for this particular manuscript, no funding was obtained.

\section{Availability of data and materials}

The datasets used and/or analyzed during the current study are available in the data repository at the date center of the EPHI. It is also available with the corresponding author on a reasonable request.

\section{Ethics approval and consent to participate}

The study used secondary data that didn't require ethical clearance. However, the SARA 2018 survey has received ethical clearance from the Institutional Review Board of Ethiopian Public Health Institute. Besides, we used summary data without a facility identifier to ensure confidentiality.

\section{Consent for publication}

Not applicable.

\section{Competing interests}

The corresponding author declares that there is no financial or non-financial competing interest.

\section{Author details}

'Reproductive Health Research Team, Ethiopian Public Health Institute, Addis Ababa, Ethiopia. ${ }^{2}$ Department of Epidemiology and Biostatistics, University of Gondar, College of Medicine and Health Science, Institute of Public health, Gondar, Ethiopia. ${ }^{3}$ Health System Research Team, Ethiopian Public Health Institute, Addis Ababa, Ethiopia. ${ }^{4}$ College of Medicine and Health Science, Institute of Public health, University of Gondar, Gondar, Ethiopia. ${ }^{5}$ Health System and Reproductive Health Research Directorate, Ethiopian Public Health Institute, Addis Ababa, Ethiopia.

Received: 21 December 2018 Accepted: 27 May 2020 Published online: 01 June 2020

\section{References}

1. Lincetto O, Mothebesoane-anoh S, Gomez P, Munjanja S. Antenatal Care: Opprotunities for Africa's Newborns. Int J Sci Technol Res.2013;2(2):51-62.

2. $\mathrm{WHO}$. WHO recommendations on antenatal care for a positive pregnancy experiance [Internet]. Luxembourg; 2016. www.who.int/reproductivehealth/ publications/maternal_perinatal_health/anc-positive-pregnancy-experience/ en/.

3. Birmeta K, Dibaba Y, Woldeyohannes D. Determinants of maternal health care utilization in Holeta town, central Ethiopia. BMC Health Serv Res. 2013; 13(1):256. Available from: BMC Health Services Research.

4. Iqbal S, Maqsood S, Zakar R, Zakar MZ, Fischer F. Continuum of care in maternal, newborn and child health in Pakistan: analysis of trends anddeterminants from 2006 to 2012. BMC Health Serv Res. 2017;17(1):189 http://www.bmchealthservres.biomedcentral.com/articles/10.1186/s12913-01 7-2111-9.

5. Ethiopian Public Health Institute. Services Availability and Readiness Assessment Ethiopia Service Availability and Readiness Assessment 2016Summary Report. Addis Ababa; 2016.

6. WHO U. Antenatal Care in Developing Country; Promises, achivements and missed opportunity. 2013.

7. EPHI. Services Availability and Readiness Assessment ( SARA ) 2018 Final Report [Internet]. Addis Ababa; 2018. http://www.ephi.gov.et/index.php/2 014-04-10-07-22-18/download.

8. EPHI. ETHIOPIAN Emergency Obstetric and Newborn Care (EmONC) Assessment 2016 [Internet]. Addis Ababa; 2016. http://www.ephi.gov.et/ index.php/2014-04-10-07-22-18/download.
9. Za Z, Am I, Nm B. Satisfaction among pregnant women towards antenatal care in public and private care clinics in Khartoum. Khartoum Med J. 2011; 04(02):590-5.

10. Fesseha G. Perceived Quality of Antenatal Care Service by Pregnant Women in Public and Private Health Facilities in Northern Ethiopia. Am J HealRes. 2014;2(4):146. http://www.sciencepublishinggroup.com/journal/paperinfo. aspx?journalid=656\&doi=10.11648/j.ajhr.20140204.17.

11. Fisseha G, Berhane Y, Worku A, Terefe W. Distance from health facility and mothers' perception of quality related to skilled delivery service utilizationin northern Ethiopia. Int J Womens Health. 2017;9:749-56.

12. Muchie KF. Quality of antenatal care services and completion of four or more antenatal care visits in Ethiopia: A finding based on a demographic andhealth survey. BMC Pregnancy Childbirth. 2017;17(1):1-7.

13. Boller C, Wyss K, Mtasiwa D, Tanner M. Quality and comparison of antenatal care in public and private providers in the United Republic of Tanzania. 2003;81(02).

14. Donabedian A. Evaluating the quality of medical care. Milbank Q [Internet]. 1966;44(No. 3, Pt. 2):166-203. http://www.pubmedcentral.nih.gov/ articlerender.fcgi?artid=2690293\&tool=pmcentrez\&rendertype=abstract.

15. EPHI. Ethiopia Service Provision Assessment Plus Survey Ethiopia Service Provision Assessment Plus ( ESPA + ) Survey. Addis Ababa; 2014.

16. FMoH. Ethiopian Health Sector Transformation Plan.2015/16 - 2019/20. Vol. 20, Federal Democratic Republic of Ethiopia Ministry of Health. 2015.

17. WHO. Service Availability and Readiness Assessment (SARA): An annual monitoring system for service delivery - Reference Manual, Version 2.2. Health Statistics and Information Systems. 2015. https://apps.who.int/iris/ bitstream/handle/10665/149025/WHO_HIS_HSI_2014.5_eng.pdf.

18. Kanyangarara M, Munos MK, Walker N. Quality of antenatal care service provision in health facilities across sub-Saharan Africa: Evidence from nationally representative health facility assessments. J Glob Health. 2017;7(2)

19. Tetui M, Ekirapa EK, Bua J, Mutebi A, Raymond Tweheyo PW. 1Department. Quality of Antenatal care services in eastern Uganda: implications for interventions. Pan Afr Med J. 2012:8688:1-15.

20. Onyeajam DJ, Xirasagar S, Khan MM, Hardin JW, Odutolu O. Antenatal care satisfaction in a developing country: a cross-sectional study from Nigeria. BMC Public Health. 2018;18(1):368.

21. Ejigu T, Woldie M, Kifle Y. Quality of antenatal care services at public health facilitie researchs of Bahir-Dar special zone, Northwest Ethiopia. BMC Heal Serv. 2013;13(1):443. http://www.ncbi.nlm.nih.gov/pubmed/24161007.

22. Powell-jackson T, Macleod D, Benova L, Lynch C, Campbell OMR. Series: Who cares for women? Towards a greater understanding of reproductive and maternal healthcare markets. The role of the private sector in the provision of antenatal care : a study of Demographic and Health Surveys from 46 lowand middle-income cou. Trop Med Int Heal Vol. 2015:20(2):230-9.

23. Rani M, Bonu S, Harvey S. Differentials in the quality of antenatal care in India. Int J Qual Heal Care 2008. 2018;20(1):62-71.

24. Williams J, Mpembeni R. Quality of antenatal and childbirth care in selected rural health facilities in Burkina Faso, Ghana and Tanzania: similar finding. Trop Med Int Heal. 2015;18(5):534-47.

25. Tuan T, Dung Vanthimai, Neu I, Dibley MJ. Comparative quality of private and public health services in rural Vietnam. Oxford Univ Press LSHTM. 2018; (August 2005).

26. Mundodan JM. Structural quality of antenatal clinics in the public health sector in a northern district in Kerala, India. JM al Int J Community Med Public Heal. 2015:2(4):513-9.

\section{Publisher's Note}

Springer Nature remains neutral with regard to jurisdictional claims in published maps and institutional affiliations. 